\title{
Electromagnetic Methods for UXO Discrimination
}

\author{
Kevin O’Neill and Juan Pablo Fernández
}

\begin{abstract}
The subsurface remote-sensing technology currently used in the United States for UXO decontamination is relatively crude, consisting of DC (static) magnetometry. Ultrawideband electromagnetic induction (EMI) is emerging as a technology with reasonable discrimination potential. EMI devices operate in the magneto-quasistatic (MQS) band, usually between tens of $\mathrm{Hz}$ and perhaps a couple hundred $\mathrm{kHz}$, and engage a substantially different phenomenology than that of wave electromagnetics. Over the relevant space scales, soil, fresh water, and rock are effectively lossless in the MQS regime, which encourages EMI application.

Here we review the relevant EMI physics and phenomenology and then discuss state-of-the-art EMI discrimination methods like the Standardized Excitation Approach (SEA). This can be used in signal matching to decide if an unseen target belongs to a catalogued set. It can also quickly provide many examples of realistic input to train statistical learning algorithms such as Support Vector Machines (SVM). SVMs can also use SEA parameters themselves as discriminators. Most realistic UXO-sensing scenarios are clutter limited. We examine computational upward continuation as a clutter-mitigation strategy with a rational physical basis.
\end{abstract}

\section{Key words:}

UXO, discrimination, Electromagnetic, induction, EMI, magneto-quasistatics, MQS, magnetometry, standardized excitations approach, SEA, excitation mode, pattern matching, magnetic dipole, dipole moment, magnetic diffusion, ground response, magnetic charge, statistical learning algorithm, support vector machine, SVM, slack variable, classification

Kevin O'Neill

Thayer School of Engineering, Dartmouth College and U.S. Army Corps of Engineers, ERDCCRREL, Hanover, NH 03755, U.S.A., e-mail: Kevin.A. O' Neill@dartmouth . edu

Juan Pablo Fernández

Thayer School of Engineering, Dartmouth College, Hanover, NH 03755, U.S.A., e-mail: jpfb@

dartmouth.edu 


\section{Introduction}

Surveying and cleanup of sites with potential contamination by unexploded ordnance (UXO) is an extremely high priority environmental objective in the United States, yet one that is very challenging. The problem is complicated by its sheer size (millions of hectares); hundreds, probably many hundreds of sites; diverse and often problematical geological and terrain conditions; and great diversity in the sizes and types of UXO. A comparable or larger scale problem exists at the international level, beyond military training grounds to the sites of past conflicts. The problem comes into focus when one notes that, while some very large UXO may be $10 \mathrm{~m}$ deep underground, by far most UXO are within the top meter of soil, mandating very shallow surveying. Further, we cannot yet sense what we are in fact most concerned about, namely the explosive within intact shells. Therefore we have to sense metal and only thereby characterize the object.

The first priority in UXO surveying is detection, that is, making sure that some sufficiently clear signal is obtained from essentially all UXO in the field. Unfortunately, in the service of this objective, our sensors record responses from virtually everything in the environment capable of producing a signal. Site remediators frequently excavate hundreds of objects for each UXO that is found [4]. The resulting costs are frequently prohibitive. Thus the second crucial requirement is that of discrimination. Signal anomalies identified in broader surveying must be subjected to close investigation to distinguish the nature of the responding object and to judge how likely it is to be a UXO. This chapter focuses on discrimination, as opposed to detection.

Electromagnetic sensors of some kind are currently the most logical choice with which to sense buried metallic bodies. Ground penetrating radar (GPR), while used in many applications of geophysical, environmental, and infrastructure surveying, has not generally been successful at distinguishing UXO. The combination of ground surface reflection, signal loss over depth, and signal clutter due to both metallic fragments and dielectric heterogeneities is simply too great. While holding some potential as an adjunct to other sensing modes for close discrimination, even ultrawideband (UWB), fully polarimetric GPR in the $10 \mathrm{MHz}-810 \mathrm{MHz}$ range coupled with extensive processing is still challenged in the discrimination realm $[6,14]$. The most common sensing mode by far in actual practice in the United States is static (DC) magnetometry. Magnetometers detect perturbations of the earth's field caused by ferrous objects. While relatively reliable at least as detectors of steel, magnetometers produce a rather crude picture by virtue of both current practice and inherent information content in the signals. Discrimination capability is quite limited, though progressing [16].

Between DC magnetometry and GPR lies electromagnetic induction (EMI) sensing. One may hope that EMI sensors combine the best of magnetometry and GPR. Like the former, they are immune to dielectric heterogeneities and, on our scale of observation, the ground is essentially transparent to them. State-of-the-art sensors are UWB, with frequencies of operation possibly from tens of $\mathrm{Hz}$ up to tens or, rarely, hundreds of $\mathrm{kHz}$, and some devices register vector response fields. Received 
signals have high information content, being sensitive to object distance, shape, orientation, and composition. Altogether, whether alone or in tandem with other kinds of sensors, EMI appears to offer the greatest immediate promise for discrimination of buried UXO.

\section{Relevant Electromagnetic Theory and Phenomenology}

To understand both the challenges and the potential of UWB EMI technology, it is vital to gain some grasp of the fundamental physics and phenomenology in that domain, particularly if one is approaching from what may be the more familiar realm of electromagnetic waves.

\subsection{Basic Relations; Waves vs. Diffusion and Potential Fields}

All the relevant phenomena are governed by Maxwell's equations [9, 20]. Faraday's Law pertains specifically to induction, stating that a time varying magnetic flux is linked to circulation of $\mathbf{E}$ :

$$
\boldsymbol{\nabla} \times \mathbf{E}=\left\{\begin{array}{cc}
-\frac{\partial \mathbf{B}}{\partial t} & \text { Time domain (TD) } \\
i \omega \mu \mathbf{H}\left[e^{-i \omega t}\right] & \text { Frequency domain (FD) }
\end{array}\right\},
$$

where $\mathbf{E}$ is the electric field $(\mathrm{V} / \mathrm{m})$ and $\mathbf{B}(\mathrm{T})$ is the magnetic flux density, equal to the magnetic field $\mathbf{H}(\mathrm{A} / \mathrm{m})$ times the medium magnetic permeability $\mu(\mathrm{H} / \mathrm{m})$. Integrating the normal component of this equation over a surface $S$, e.g. the planar region within the loop in Figure 1, produces an integral version of (1):

$$
\oint_{\Gamma} d \boldsymbol{\gamma} \cdot \mathbf{E}=-\frac{d}{d t} \int_{S} d S B_{n}=-\frac{d \Phi}{d t} .
$$

The line integral around the edge of the surface, $\Gamma$, constitutes essentially a voltage, an electromotive force. If there is a conductor surrounding the surface, this implies an electric current loop. Depending on what part of the system is forced, such a current loop may induce a changing magnetic flux $\Phi$, as in our EMI transmitters; or, conversely, an imposed change in magnetic flux may induce a current loop, as when our transmitted magnetic fields encounter a metallic object (see Figure 1).

An infinitesimal magnetic dipole produces an $\mathbf{H}$ field given by [20]

$$
\mathbf{H}(\mathbf{r})=\frac{3 \hat{\mathbf{r}} \hat{\mathbf{r}}-\mathbf{I}}{4 \pi r^{3}} \cdot \mathbf{m}
$$


Fig. 1 Schematic of a magnetic dipole formed by an electric current loop, with associated magnetic field lines passing through the loop.

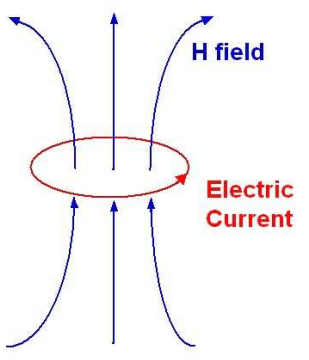

where $\mathbf{m}$ is the dipole moment of the current loop. For this ideal dipole approximation to apply, the distance $r$ from the center of the loop need only be greater than a couple times the loop diameter.

As all our EMI transmitters and responding objects of interest form finite dipoles, the relation in (3) is fundamental, at least as an approximation. In particular, the $1 / r^{3}$ spatial dependency of signals produces crucial limits on applicability and resolution of the technology. Except rarely, where noted, we will assume in what follows that the sensor's transmitters and receivers are co-located. In this case, (3) applies over the same distance, $r$, in both directions between transmitter and responding object, for a total signal falloff proportional to $1 / r^{6}$. As we shall see below, ground lossiness does not afflict EMI with signal loss in the same way that it does GPR, where it is a serious problem. Instead, the $1 / r^{6}$ signal falloff in EMI is due purely to the inherent geometry of the quasistatic fields. Thus there is little that can be applied to counteract it.

Ampère's Law relates the curl of the magnetic field to various currents:

$$
\boldsymbol{\nabla} \times \mathbf{H}=\left\{\begin{array}{c}
{\left[\mathbf{J}_{\mathrm{sc}}\right]+\mathbf{J}_{\mathrm{env}}+\frac{\partial \mathbf{D}}{\partial t}} \\
{\left[\mathbf{J}_{\mathrm{sc}}\right]+\sigma \mathbf{E}-i \omega \varepsilon \mathbf{E}}
\end{array}\right\} .
$$

The source current $\mathbf{J}_{\mathrm{sc}}$ is taken to be non-zero only in isolated, concentrated regions (e.g., a wire loop as in Figure 1), and we do not analyze it further. The conduction current density in the environment, $\mathbf{J}_{\text {env }}\left(\mathrm{A} / \mathrm{m}^{2}\right)$, is related to the electric field via the electrical conductivity $\sigma(\mathrm{S} / \mathrm{m})$; and $\partial \mathbf{D} / \partial t$ is the "displacement current," where $\mathbf{D}=\varepsilon \mathbf{E}$ and $\varepsilon$ is the permittivity of the medium $(\mathrm{F} / \mathrm{m})$. The exposition that follows explores the ways in which the magnitudes of the last two terms on the right, relative to one another and to the quantity on the left, fundamentally determine the nature of the electromagnetic phenomena at hand.

The divergence law for magnetic fields states, in effect, that there are no isolated magnetic charges (poles):

$$
\boldsymbol{\nabla} \cdot \mathbf{B}=0=\boldsymbol{\nabla} \cdot \mu \mathbf{H}
$$


In magnetically homogeneous regions, $\mathbf{H}$ will be divergence-free as well. Equation (5) also means that the magnetic field lines in Figure 1 actually form closed loops. As a convenient fiction, we may introduce nonzero equivalent magnetic charges $q_{m}$ on the right in (5), outside of regions of application, in order to generate mathematically the fields within regions of interest (ROI). This is valid as long as the source distributions imply fields that satisfy the governing equations within the ROI as well as the appropriate conditions on its boundary. A $q_{m}$-based approach is used in the clutter-suppressing upward continuation system described below.

Using (5) for $\mathbf{H}$ together with (1) in the curl of (4) produces a Helmholtz-type equation:

$$
\nabla^{2} \mathbf{H}=\left\{\begin{array}{cc}
\sigma \mu \frac{\partial \mathbf{H}}{\partial t}+\mu \varepsilon \frac{\partial^{2} \mathbf{H}}{\partial t^{2}} & (\mathrm{TD}) \\
\left(i \omega \sigma \mu+\omega^{2} \mu \varepsilon\right) \mathbf{H}=-k^{2} \mathbf{H} & (\mathrm{FD})
\end{array}\right\},
$$

where $k$ has different meanings depending on the parameter range that applies. When the second derivative with respect to time dominates then (6) becomes a wave equation, possibly with a significant loss term. In that case, $k$ is a true wave number. When the first derivative with respect to time dominates, then (6) becomes a diffusion equation and $k$ is no longer a true wave number.

The relative magnitude of the two time-derivative terms in (6) is probably best apprehended from the ratio of their corresponding frequency-domain expressions, namely $\sigma / \omega \varepsilon$. In the GPR frequency range $\left(10^{7}-10^{9} \mathrm{~Hz}\right)$ we may assume that the fields form waves in air $(\sigma \sim 0)$ and that penetration of metallic reflectors is negligible. They serve as perfect reflectors. The dielectric constant of soil, $\kappa=\varepsilon / \varepsilon_{0}$, ranges from about 6 for dry soil to a maximum of about 30 for soil completely saturated with water. For ground, $\sigma$ ranges from a low of about $10^{-3} \mathrm{~S} / \mathrm{m}$ (particularly for dry and granular soil) up to about $1 \mathrm{~S} / \mathrm{m}$ for media saturated with seawater. Altogether, for GPR we have

$$
\frac{\text { Diffusion }}{\text { Wave }} \sim \frac{\sigma}{\omega \varepsilon}=\left\{\begin{array}{ll}
10^{-3}, & \text { lowest } \sigma, \text { highest } f \\
10^{2}, & \text { highest } \sigma, \text { lowest } f
\end{array} .\right.
$$

In principle, either waves or diffusion may dominate. In the absence of saltwater in the soil, a more typical maximum of the ratio in (7) is on the order of unity. Thus wave phenomena are typically dominant or at least highly significant.

Magneto-quasistatics is defined by the condition that the displacement current $\partial \mathbf{D} / \partial t$ is negligible. This can occur when the $\varepsilon$ term on the right hand side of (6) is overshadowed by the $\sigma$ term. It is the case over essentially the entire EMI band in soil and metal.

$$
\frac{\text { Diffusion }}{\text { Wave }} \sim \frac{\sigma}{\omega \varepsilon}=\left\{\begin{array}{l}
\text { Soil } \Rightarrow\left\{\begin{array}{l}
\sim 10^{1}, \text { lowest } \sigma \text {, highest } f \\
\sim 10^{8}, \text { highest } \sigma \text {, lowest } f
\end{array}\right\} \\
\text { Metal } \quad \sigma \sim 10^{7} \mathrm{~S} / \mathrm{m}, \quad \Rightarrow \quad \sigma / \omega \varepsilon \gg 1
\end{array}\right.
$$


Fig. 2 Skin depth vs. frequency for various common metals over the EMI band.

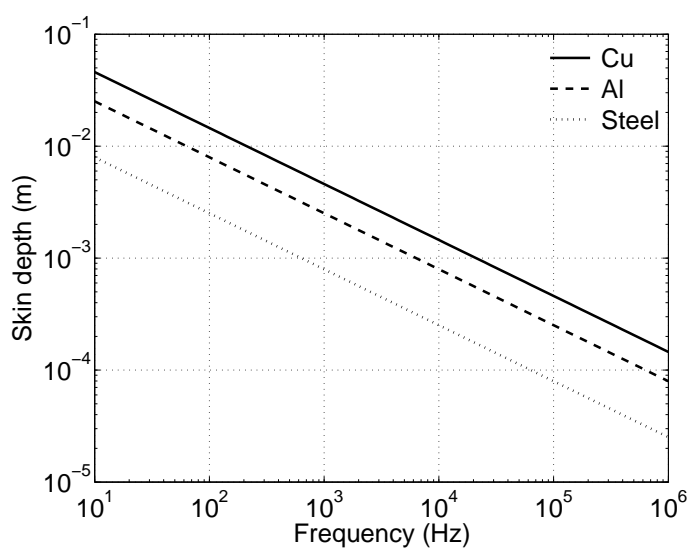

While diffusion can be very important, waves are essentially always negligible. In the EMI realm there are no true reflections, diffractions, resonances, etc., of the sort expected in the wave regime.

As explained below, it is possible for both terms on the right hand side of (4) to be negligible relative to the derivatives in the term on the left. In this case, the magnetic field is irrotational and can be represented simply in terms of the gradient of a scalar potential $\Psi[9,20]$ :

$$
\boldsymbol{\nabla} \times \mathbf{H}=0 \quad \longrightarrow \quad \mathbf{H}=-\boldsymbol{\nabla} \Psi .
$$

Combining this with the divergence-free condition on $\mathbf{H}$ produces a simple Laplace governing equation in terms of $\Psi$ :

$$
\boldsymbol{\nabla} \cdot \mathbf{H}=0 \quad \longrightarrow \quad \nabla^{2} \Psi=0
$$

A scalar potential may be generated most simply via scalar sources, i.e. equivalent magnetic charges $q_{m}$ situated outside the ROI within which the divergence free condition applies to $\mathbf{H}$ :

$$
\Psi(\mathbf{r})=\int_{S_{0}} d S^{\prime} \frac{q_{m}\left(\mathbf{r}^{\prime}\right)}{4 \pi\left|\mathbf{r}-\mathbf{r}^{\prime}\right|} .
$$

By construction, $\Psi$ obtained from (11) from any set of $q_{m}$ outside the ROI will satisfy the equations in $\mathbf{H}$ within the ROI, as per (9) and (10). To obtain the particular, realistic field required in any circumstance, one uses the gradient of (11) to enforce the standard boundary conditions in $\mathbf{H}[9,20]$ on the boundary of the ROI. This is the strategy we will employ for upward continuation. 


\subsection{Character of the EMI Regime: Metal}

Metallic targets are significantly penetrable over much of the EMI band (Figure 2). From the point of view of discrimination of unknown targets, this is both fortunate and unfortunate: metal type matters. Figure 3, left, shows the frequency response to a uniform excitation $\mathbf{H}$ field by a hypothetical $20-\mathrm{cm}$ diameter metal sphere, a case for which there is an analytic solution [23]. The response is construed in terms of a component in phase with the excitation field (real-valued part) and a part in phase quadrature with it (imaginary part). Around the high-frequency limit, penetration of the excitation field is so slight that essentially only surface currents exist and asymptotic behavior is reached, independent of material type. In accordance with Lenz's law, these surface currents circulate in such a way as to oppose the primary field, hence the negative real value of the response there.

At the low-frequency end of the spectrum, approaching magnetostatic conditions, penetration of the object by the excitation field is complete but there are no induced currents because $\partial \mathbf{B} / \partial t$ is negligible. If the metal is permeable $\left(\mu>\mu_{0}\right)$, as in the example in the figure, a magnetization (polarization) response appears in the absence of macroscopic induced currents. It is aligned with the excitation field (positive sign) and is due to microscopic magnetic dipole structures within the material. For non-permeable materials $\left(\mu=\mu_{0}\right)$, the inphase response will be zero at the low-frequency limit, descending from there toward the high-frequency asymptote. Between the low- and high-frequency regimes, over most of the band, one encounters some mixture of magnetization and induced (macroscopic) current responses within the object. The quadrature component is due to induced volume currents and is delayed relative to the excitation field because of the finite conductivity of the material. This effect peaks somewhere within the band.

Given that $\mathbf{D}$ and its derivatives are negligible in magneto-quasistatic fields, one can manipulate the equations above to show that induced currents must be divergence-free. There can be no accumulations of free charge, and all induced currents must form continuous, closed loops. The current loops induced by impinging primary (excitation) fields thus effectively form finite-dimensional magnetic dipole structures. For the special case of a homogeneous sphere, the induced currents and polarization throughout its volume produce a secondary field outside the object that is exactly the same as would be produced by an infinitesimal magnetic dipole at its center.

While essentially all metallic objects produce some variant of the relaxation-type curves in Figure 3, left, the particulars are case-dependent. Material type, size, proportions, and orientation all influence the location of the quadrature peak as well as many specific details in the relations between the two components. Figure 3, right, shows normalized quadrature response spectra of a hypothetical steel prolate spheroid, $20 \mathrm{~cm}$ by $5 \mathrm{~cm}$, with the same material parameters as the sphere on the left and with its axis oriented parallel and then perpendicular to the excitation field. A new analytical solution is available for these cases [1]. Note that the peak in this component shifts much higher in frequency for the transverse orientation. 

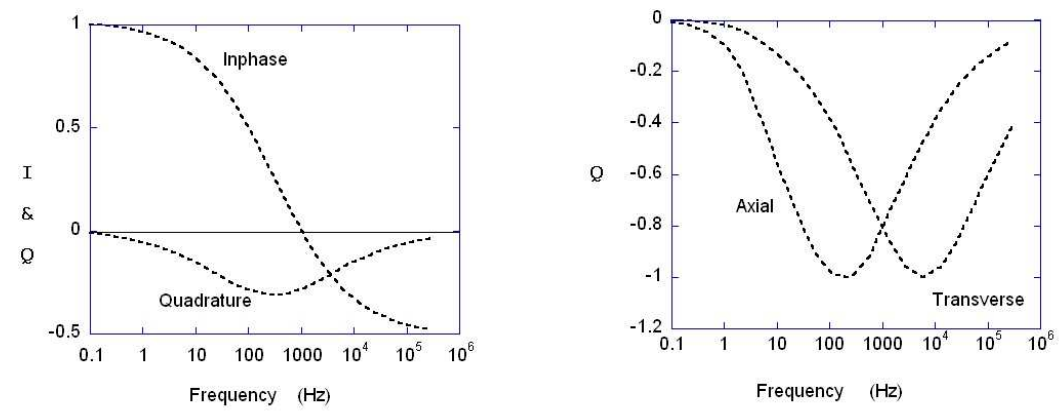

Fig. 3 Left: Frequency response of a sphere with properties of steel, $\sigma=4 \times 10^{6} \mathrm{~S} / \mathrm{m}, \mu_{r}=100$. Right: normalized transverse and axial responses by a prolate spheroid of the same material.

Examining spectral features such as this is the basis for frequency-domain (FD) discrimination.

These spectral features also correspond to response patterns in time when the object is subjected to an imposed change in the surrounding magnetic field. Responses to any such excitations can only decay through the time following the change, but with patterns of magnitude, temporal gradient, etc., dependent on the object's particulars. Time-domain (TD) discrimination examines the features of such received temporal decay curves. Note that, while responses proceed through time in TD EMI sensing, signal time does not correspond to distance to a responding entity, as in radar. On our EMI time and space scales there is no true wave-type propagation, and all distances sensed respond effectively at the same time.

\subsection{Character of the EMI Regime: Air}

One can show that the magnitude of $\partial \mathbf{D} / \partial t$ in air in the EMI band is negligible compared to the terms in $\boldsymbol{\nabla} \times \mathbf{H}$. Because the electrical conductivity of air is also negligible, neither displacement nor conduction currents are significant, and the right hand side of (4) is zero. With an irrotational $\mathbf{H}$ field, the simple scalar Laplace equation (10) governs. Viewed another way, as long as there is time variation of the fields, they are waves on some scale, but not on our scale of observation $(\sim 1 \mathrm{~m})$. Wavelengths range from some kilometers at the top of the band up to perhaps $10,000 \mathrm{~km}$ near the bottom. Over our scale of observation there is no discernible phase difference between one point and another; no delay. The fields have the structure of static fields with time dependence imparted only by boundary conditions/forcing functions. 
Fig. 4 Skin depths for different soil conductivities, assuming a representative dielectric constant of 16 .

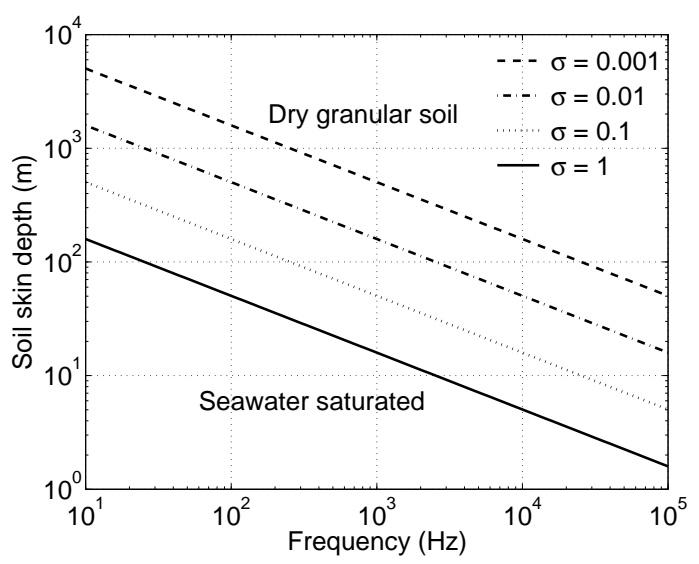

\subsection{The EMI Regime in Soil}

Here $\sigma$ is finite and one can show that conduction currents will typically have a larger effect than displacement currents. At the same time, the effect of $\sigma$ is negligible: Skin depths are much greater than the scale of observation (Figure 4). In this sense, the soil is transparent to signals in the EMI band. In the absence of seawater, the upper two lines probably furnish reasonable bounds under common circumstances. With induced soil currents negligible and displacement currents even smaller, once again the $\mathbf{H}$ field is irrotational and a scalar Laplace potential equation governs. Further, note that as tangential $\mathbf{E}$ fields are continuous between soil and buried metal objects, the ratio of induced current magnitudes in each will be approximately the ratio of their conductivities, which is $\sim 10^{9}$. Currents induced in the metal dominate the response signal within the soil, given that we only sense a soil volume that is not many orders of magnitude greater than that of a target of interest. Of course, this situation may not be the case in the absence of metal targets and when large volumes or depths of ground are sensed, e.g. $\sim \mathrm{kms}$ in deep geophysical prospecting. Overall, in our case, for all practical purposes the transmitted field reaches a metallic buried object essentially unaffected by the ground, and system responses both within the soil and above ground at the receiver are dominated by the response of the target.

While induced soil currents do not produce significant signal responses, magnetically permeable soil can still, however, produce a significant half-space response, including rough surface effect, which we indeed see in field work. The relative permeability $\mu_{r}$ is unity in free space; for soil it is typically construed in terms of the (volumetric) magnetic susceptibility as $(1+\chi)$. Even though $\chi$ magnitudes are generally on the order of $10^{-3}$ or less [4], this can still be enough to produce a half-space response that is notable relative to that of buried metal targets. Some UXO sites are particularly problematic, such as volcanic terrains (e.g., Maui and Kaho'olawe in Hawai ${ }^{i}$ ). The nature and character of soil susceptibility are currently an area of 
active research. Our recent experience suggests that, for common soil, the instantaneous response (real-valued $\chi$, constant over the band) is large relative to a trailing relaxation response, with only the latter affecting TD instruments [26].

\subsection{The EMI Realm, Summary}

In the MQS EMI band, on our scale of observation:

- There are no waves nor attendant wave phenomena (reflection, diffraction, refraction...). To emphasize the difference, we speak of the transmitted field impinging on a target as the "primary" (as opposed to "incident") field, and the field from the object's response as the "secondary" field.

- One can map the vector, UWB subsurface response over an area of ground surface in terms of inphase and quadrature components in the FD, overall using perhaps five decades of frequency, or using decay time points in the TD.

- Metal targets are penetrable, completely so at the very bottom of the band, with possibly negligible penetration (surface currents only) at the very top of the band. Different metal types respond differently, and the magnetic field within the metal operates by diffusion.

- In both soil and air, both conduction and displacement currents, i.e., both diffusion and wave effects, are negligible. Magnetic fields are irrotational and can be expressed in terms of a scalar Laplacian potential. The only significant induced currents are those in metal objects, which dominate the secondary fields in the soil and air in their vicinity.

- In contrast to GPR, there is no significant delay, travel time, or phase difference over space-fields have the structure of magnetostatic fields, with time dependence imposed by boundary conditions/forcing functions. In TD EMI, elapsed signal time corresponds to duration of signal decay in an object at a given depth. It does not correspond to depth of responding entities. All depths respond essentially at once and a single picture emerges over each portion of the ground surface.

- Soil lossiness has a negligible effect. In that sense the ground is transparent to EMI signals, though half-space-type magnetic responses from permeable soils are seen.

\section{Standardized Excitation Approach Forward Modeling in EMI}

For use in many kinds of discrimination algorithms, we benefit from rigorous but fast simulations of EMI responses by objects of interest, taking into account the particular sensor characteristics. The Standardized Excitation Approach (SEA) formulation based on fundamental spheroidal modes can calculate the sensor responses produced by a geometrically complex, materially heterogeneous object, accounting 
Fig. 5 Schematic of a radar beam incident upon a UXO, indicating the beam's decomposition into constituent plane waves.

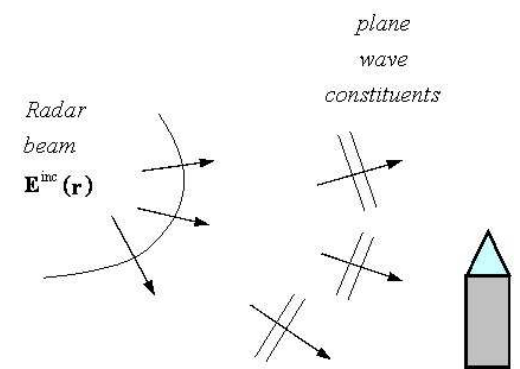

for near and far field effects and all internal interactions [7, 19, 21, 25]. The total response to any excitation is constructed simply as an appropriate superposition of responses to defined excitation modes. These modes form a sufficient basis to express any excitation. As the simulations are extremely fast relative to detailed numerical solution of the governing equations, they can be run many times in the course of an inversion or classification computation.

Consider an analogy to decomposition of radar beams into constituent plane waves (Figure 5). At each frequency, an incident radar beam can be decomposed into a bundle of plane waves, all of the same frequency but with different vector wave numbers $\mathbf{k}_{j}$ depending on the direction of propagation of each:

$$
\mathbf{E}^{\mathrm{inc}}(\mathbf{r})=\sum_{j} \beta_{j} \mathbf{E}_{j}^{\mathrm{inc}}(\mathbf{r}), \quad \mathbf{E}_{j}^{\mathrm{inc}}(\mathbf{r})=e^{i \mathbf{k}_{j} \cdot \mathbf{r}} .
$$

If, either by computation or experiment on a particular object, one catalogues its response to a unit magnitude of each of these constituent $\mathbf{E}_{j}^{\text {inc }}$, then one can easily construct the total response to the bundle of them constituting the complete beam. One can do this for any sensor-target arrangement as quickly as one can solve for the $\beta_{j}$ in (12).

To parallel this procedure in EMI, for magnetic fields, one decomposes the primary field into fundamental or "standardized" excitations,

$$
\mathbf{H}^{\mathrm{PR}}(\mathbf{r})=\sum_{j} b_{j} \mathbf{H}_{j}^{\mathrm{PR}}(\mathbf{r})
$$

and constructs the total response $\mathbf{H}^{s}$ as the corresponding sum of responses to each excitation mode:

$$
\mathbf{H}_{j}^{\mathrm{PR}}(\mathbf{r}) \rightarrow \mathbf{H}_{j}^{s}(\mathbf{r}), \quad \mathbf{H}^{s}(\mathbf{r})=\sum_{j} b_{j} \mathbf{H}_{j}^{s}(\mathbf{r})
$$

Cataloguing the response to each excitation mode means solving for some set of parameters, $S_{k}^{j}$, for each fundamental ( $j$ th) input, i.e., setting $\mathbf{H}_{j}^{s}(\mathbf{r})=\sum_{k} S_{k}^{j} \mathbf{G}_{k}(\mathbf{r})$. One can obtain the $S_{k}^{j}$ from data in controlled measurements on an object of interest by calculating the $b_{j}$ for various sensor-object configurations via (13), then using these in the combination of (13) and (14): 


$$
\mathbf{H}^{s}(\mathbf{r})=\sum_{j} b_{j} \sum_{k} S_{k}^{j} \mathbf{G}_{k}(\mathbf{r})
$$

With the $b_{j}$ known and a sufficient number of measurements of the left-hand side of (15), one can solve for the necessary $S_{k}^{j}$. For a given object or object type, this need only be done once. While the data or particular beam composition may be a function of the sensor position $\mathbf{r}$, the $S_{k}^{j}$ are not. When their nature or structure is defined, they are invariant characteristics of the object.

The nature of the modal response function $\mathbf{G}_{k}(\mathbf{r})$ just depends on the nature of the response parameters $S_{k}^{j}$ that are applied. For example, if the $S_{k}^{j}$ are equivalent charges at the object location, then the $\mathbf{G}_{k}(\mathbf{r})$ will just be the appropriate Green function for that source type, see e.g. (11). The $S_{k}^{j}$ can be used thereafter for repeated forward modeling, requiring only that one decompose any excitation at hand, i.e. obtain the applicable $b_{j}$ for each sensor-object configuration. As we shall see, having obtained the characteristic $S_{k}^{j}$ for candidate objects, one can use them within fast forward models during optimization to determine whether recorded signals are most likely to have been produced by one of the candidates. Alternatively, one can infer the $S_{k}^{j}$ for unknown targets and then use those parameters themselves as discriminators.

The fundamental problem in formulating the SEA approach in EMI resides in the requirement that one produce some appropriate basis $\mathbf{H}_{j}^{\mathrm{PR}}(\mathbf{r})$ for decomposing the primary fields. In the EMI/MQS regime, there are no plane waves nor, for that matter, any waves at all. One solution is to apply basic solutions of the Laplace equation in the spheroidal coordinate system,

$$
\begin{aligned}
\mathbf{H}^{\mathrm{PR}}(\eta, \xi, \phi) & =-\sum_{m, n} b_{m, n} \boldsymbol{\nabla}\left(P_{n}^{m}(\eta) P_{n}^{m}(\xi)\left\{\begin{array}{c}
\sin m \phi \\
\cos m \phi
\end{array}\right\}\right) \\
& =-\sum_{j} b_{j} \nabla \psi_{j}^{\mathrm{PR}}(\eta, \xi, \phi),
\end{aligned}
$$

where $P_{n}^{m}$ is the associated Legendre function of the first kind of order $m$ and degree $n$, and $j$ denotes admissible combinations of $m$ and $n$ ([1] and references therein). We frequently choose prolate spheroidal systems, with origin at the (possibly hypothetical) object location, because UXO typically have elongated, rotationally symmetric shapes, requiring few terms in the series. Figure 6 shows example magnetic field lines for some of the lowest modes in the series in (16).

Instead of using responses by equivalent sources (e.g. charges), one can construct the response to each fundamental excitation using an analytic spheroidal function series, similar to the one expressing the primary field:

$$
\begin{aligned}
\mathbf{H}_{j}^{s}(\eta, \xi, \phi) & =-\sum_{m, n} B_{m, n}^{j} \boldsymbol{\nabla}\left(P_{n}^{m}(\eta) Q_{n}^{m}(\xi)\left\{\begin{array}{c}
\sin m \phi \\
\cos m \phi
\end{array}\right\}\right) \\
& =-\sum_{k} B_{k}^{j} \boldsymbol{\nabla} \psi_{j}^{s}(\eta, \xi, \phi),
\end{aligned}
$$



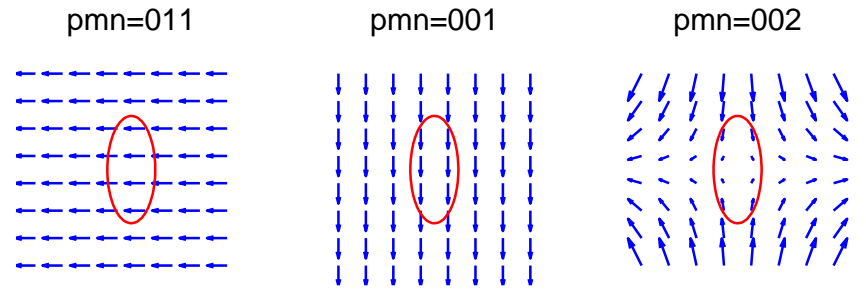

$\mathrm{pmn}=012$

Fig. 6 Any primary field can be considered as the superposition of a set of predefined spheroidal excitation modes $\mathbf{H}_{j}^{\mathrm{PR}}(\mathbf{r})$. Here we see the magnetic field lines corresponding to some of the most fundamental modes.

Fig. 7 The most significant coefficients in the decomposition of an example EMI primary field.

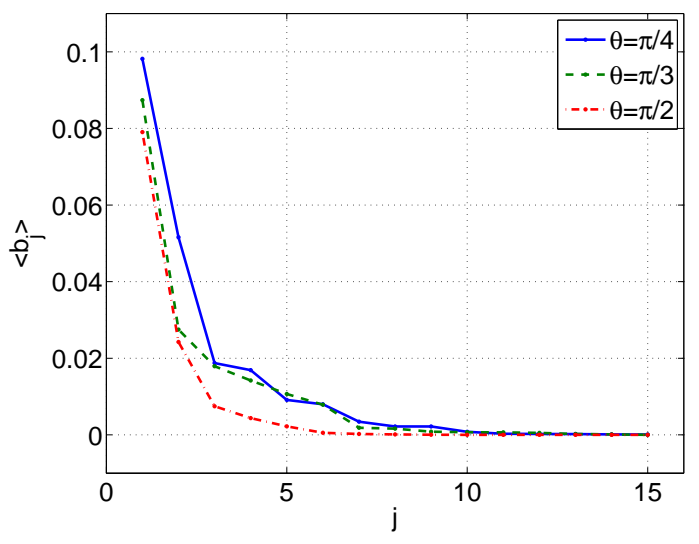

where $Q_{n}^{m}$ is the associated Legendre function of the second kind of order $m$ and degree $n$. In this case, the $S_{k}^{j}$ in (15) are just the coefficients in the series in (17), i.e. the $B_{k}^{j}$. While this formulation presents its own difficulties relative to a source-based response parameterization, it is distinguished by the fact that the $B_{k}^{j}$ are unique. That is, one can show that they are characteristics of the object, regardless of excitation or manner of observation, and any object possesses one and only one set of them in a chosen coordinate system $[7,8]$.

A crucial feature of EMI SEA decomposition using spheroidal potential functions is that very few of them are required. Figure 7 shows averages over instrument position of the $b_{j}$ values obtained experimentally for a particular UXO, using a relatively small FD sensor. The sensor-UXO separation was about one to two characteristic lengths relative to both sensor and target. Also, this sensor produces rather nonuniform primary fields relative to most other instruments. Even so, only about four modes dominate the primary field distribution around the UXO. Figure 8 shows a test in which the UXO response predicted by the SEA is compared to measurements, using either 4 or 8 excitation modes. The measurements proceed in sweeps along the grid lines, producing peaks in the signal as the sensor moves past the object. The plots on the right illustrate the character of the overall results. The 8-mode 
formulation produces slightly more accurate results than the 4-mode one; however, the difference is not great and probably not justified on the basis of cost vs. benefit. The reader is referred to the literature $[8,12,19,21,24,25]$ for discussion of alternatives in pursuing the modal parameters. Issues include control of possible ill-conditioning when one seeks to use higher modes or those only marginally supported by the data.

The SEA model of the same UXO was also used in an optimization over position and orientation to determine the best match (lowest mismatch) it can produce with each recorded signal from a collection of objects. These consisted of machined metal spheroids, combinations of spheroids, and the UXO (Figure 9). The SEA rendering of the UXO indeed produces the lowest mismatch to the actual UXO signal.

Further developments of the SEA include the Normalized Surface Magnetic Charge (NSMC) formulation [18]. The NSMC uses a particularly simple breakdown of the primary field together with synthesis of responses via connected equivalent source distributions. The integral of the source mechanisms itself furnishes a distinctive characterization of the object.

\section{Support Vector Machines and their Application}

A possible way to avoid time-consuming nonlinear searches during UXO discrimination can be to perform "before the fact" inversion. One can run a trustworthy model very many times to generate artificial data representative of the expected parameter space. An algorithm could then take those results, make sense of them by weighing the available empirical evidence without any reference to the underlying model, and apply this knowledge to make predictions about unseen cases. In this section we describe one such method, the Support Vector Machine (SVM) [2, 10]. We describe how an SVM can perform binary classification, a task to which most classification and regression problems can be reduced, and then show the results of some SVM experiments related to UXO discrimination.

The "examples" from which an SVM learns to classify are a set $\left\{\mathbf{x}_{i}\right\}$ of $n$ dimensional vectors. In the UXO problem these can be raw measured fields or distilled parameters-dipole moments or spheroidal expansion coefficients, for example-expected to contain evidence of the character of an object. Depending on the classification we want to make we assign a yes/no attribute to each point: examples belonging to the desired class have $y_{i}=1$ and the others $y_{i}=-1$. SVMs carry out the classification by finding a linear surface, a hyperplane, that divides the parameter space into two distinct regions, each of which hopefully contains points from only one of the categories (Figure 10). During the learning process the machine readjusts the hyperplane parameters to accommodate every training vector until it reaches an optimal compromise. At that point, only those examples whose removal would significantly change the locus of the hyperplane suffice to specify a predicting function. These points with high information content are the support vectors that give the method its name. 

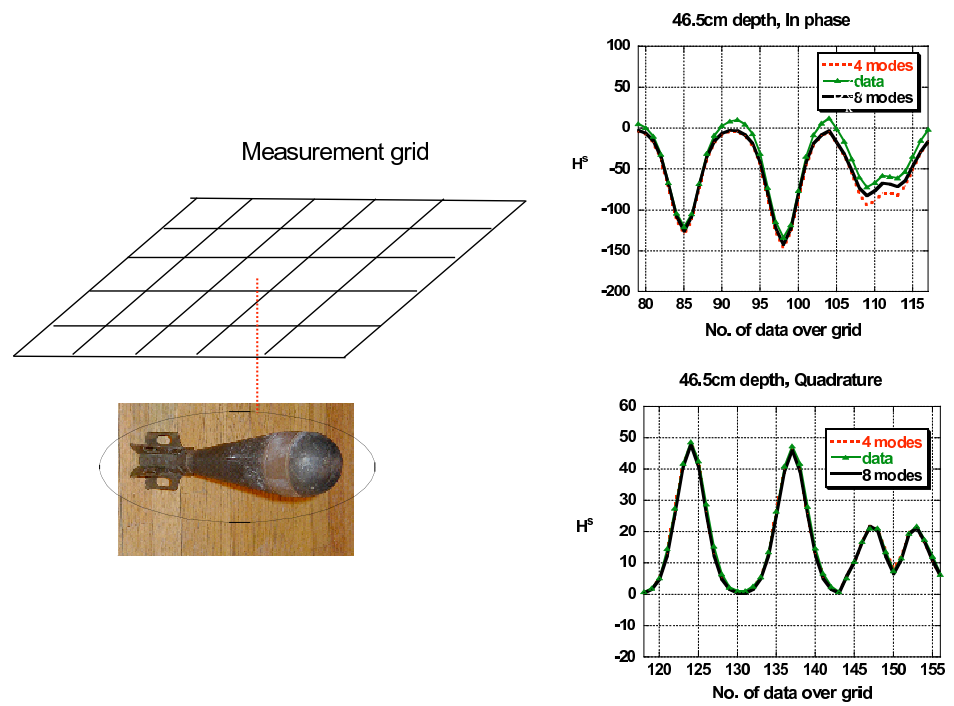

Fig. 8 SEA parameters for a model of the UXO are obtained from controlled measurements over the grid, then used (plots on right) to predict the signal at other elevations of the sensor. The results using 8 excitation modes show only slight benefits from inclusion of the higher-order coefficients.

\begin{tabular}{c|cccc}
\hline Object & Material & Axis (2a) & Axis $(2 b)$ & $e=b / a$ \\
\hline \hline S2 & Steel & $30 \mathrm{~mm}$ & $182 \mathrm{~mm}$ & 6 \\
S3 & Steel & $30 \mathrm{~mm}$ & $90 \mathrm{~mm}$ & 3 \\
S4 & Steel & $15 \mathrm{~mm}$ & $90 \mathrm{~mm}$ & 6 \\
S7 & Steel & $30 \mathrm{~mm}$ & $30 \mathrm{~mm}$ & 1 \\
A2 & Aluminum & $30 \mathrm{~mm}$ & $91 \mathrm{~mm}$ & 3 \\
A3 & Aluminum & $15 \mathrm{~mm}$ & $91 \mathrm{~mm}$ & 6 \\
\hline C1 & \multicolumn{4}{|c}{ S4; S7 } \\
C2 & \multicolumn{4}{|c}{ A3; S7 } \\
\hline U1 & \multicolumn{4}{|c}{ UXO of Figure 8, mainly steel } \\
\hline
\end{tabular}

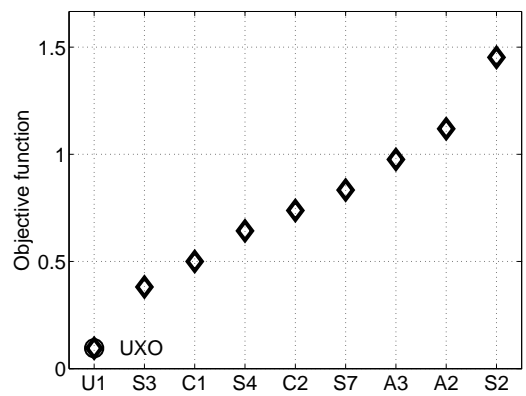

Fig. 9 In a signal pattern matching test over the grid, optimization using the SEA model of the mortar indicates correctly that the model is capable of producing the best match when the UXO in fact produced the data, as opposed to the other objects. The other items produce lowest mismatches that are roughly an order of magnitude worse [24]. 
Fig. 10 Support vector classification. The weight vector is perpendicular to the separating hyperplane. The negative of the bias divided by the norm of the weight is the separation between the hyperplane and the origin. The support vectors are circled.
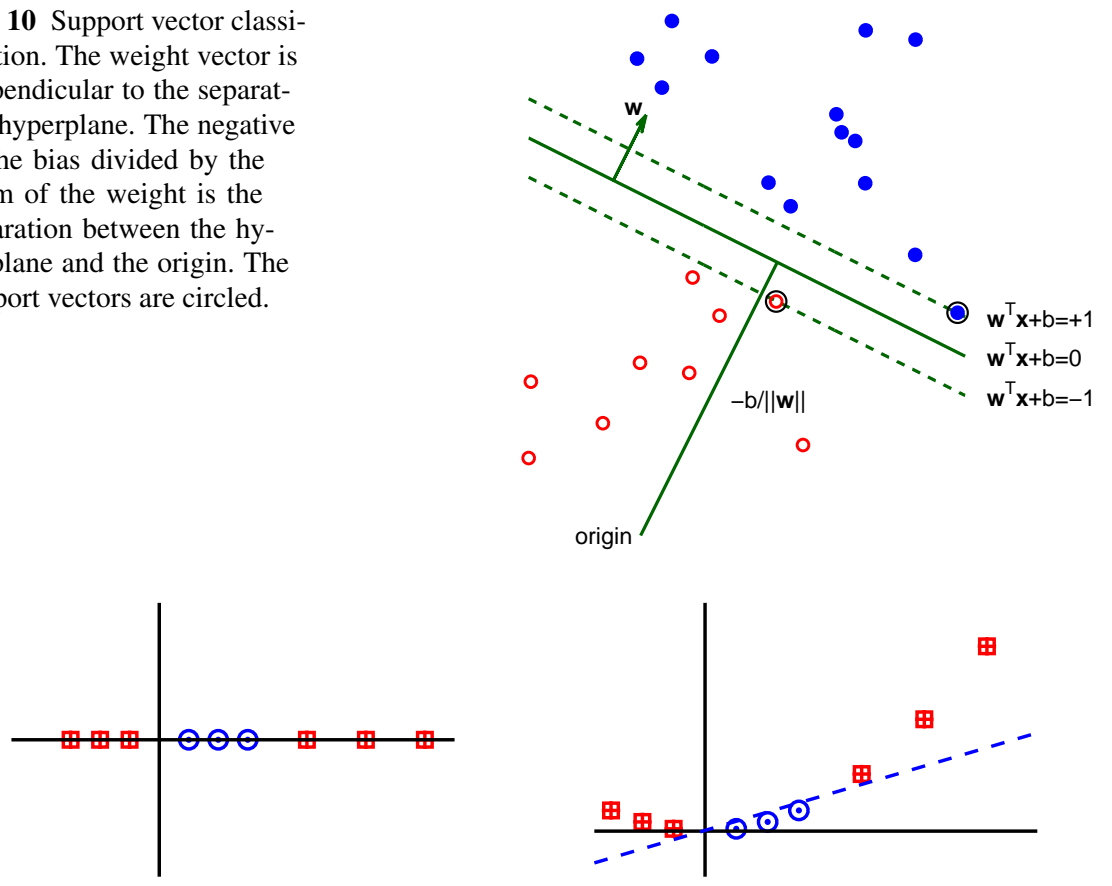

Fig. 11 Applying a polynomial kernel to a nonseparable set of points projects it into a higherdimensional space and results in a linearly separable distribution. Left: original data in $X$, with two classes corresponding to the circles and squares. Right: data projected into $(X, Y, Z)=\left(x \sqrt{ } 2, x^{2}, 1\right)$, shown on the $X-Y$ plane.

Most data sets are not linearly separable in the space they occupy, and even bona fide separable sets may be corrupted into nonseparability by noise. On the other hand, it should be possible to make any set separable by projecting it into a space of high enough dimensionality. The separating surface would be flat by construction in the new space but could be curved-even multiply connected—in the original. However, there must be a means to limit the capacity of the machine, its ability to classify any data set without error: a machine with too much capacity is like a model with too many adjustable parameters in its tendency to overfit data and noise and concentrate on details rather than on essentials. We must be willing to tolerate some mistakes if we want to generalize well, and the SVM algorithm incorporates this in a transparent way [3].

A hyperplane in $n$ dimensions is completely described by the equation

$$
\mathbf{w}^{\mathrm{T}} \mathbf{x}+b=0,
$$

where the weight vector $\mathbf{w}$ is perpendicular to it and the scalar bias $b$ is proportional to its separation from the origin (see Figure 10). Knowing $\mathbf{w}$ and $b$ the machine classifies any subsequent example $\mathbf{z}$ by evaluating $f(\mathbf{z})=\operatorname{sgn}\left(\mathbf{w}^{\mathrm{T}} \mathbf{z}+b\right)$. 
Statistical learning theory [22] proves that the hyperplane that minimizes a properly defined "expected generalization error" for a given set of points is that with the smallest norm [2]. An SVM sets out to solve the constrained minimization problem

$$
\begin{aligned}
\min _{\mathbf{w}, b} & \frac{1}{2} \mathbf{w}^{\mathrm{T}} \mathbf{w} \\
\text { s.t. } & \left(\mathbf{x}_{i}^{\mathrm{T}} \mathbf{w}+b\right) y_{i} \geq 1 .
\end{aligned}
$$

To prevent overfitting, we relax the constraints by introducing slack variables that measure how far a point strays into the "wrong" side:

$$
\begin{aligned}
\min _{\mathbf{w}, b} & \frac{1}{2} \mathbf{w}^{\mathrm{T}} \mathbf{w}+C \sum_{i} \xi_{i} \\
\text { s.t. } & \left(\mathbf{x}_{i}^{\mathrm{T}} \mathbf{w}+b\right) y_{i} \geq 1-\xi_{i}, \quad \xi_{i} \geq 0 .
\end{aligned}
$$

Note that we also penalize the objective function for each misclassified example; the proportionality constant $C$ is the capacity we referred to above.

It is more convenient to solve this problem in its dual formulation. To paraphrase an introductory calculus problem, instead of finding the rectangle with minimum perimeter when given its area we will fix the perimeter and look for the rectangle with maximum area. Both problems have the same answer, but the second involves a simpler constraint and is easier to solve; this advantage is amplified in the multidimensional problem (19), whose inequality constraints become equalities [10]:

$$
\begin{array}{cl}
\max _{\boldsymbol{\alpha}} & \sum_{i} \alpha_{i}-\frac{1}{2} \sum_{i, j} \alpha_{i} y_{i} \mathbf{x}_{i}^{\mathrm{T}} \mathbf{x}_{j} y_{j} \alpha_{j} \\
\text { s.t. } & \sum_{i} \alpha_{i} y_{i}=0, \quad 0 \leq \alpha_{i} \leq C .
\end{array}
$$

The solution to the new problem is a vector of Lagrange multipliers $\alpha_{i}$, each of which in a sense measures the information content of its corresponding point. Only a small fraction of the examples, the support vectors, have nonzero $\alpha_{i}$. Note that in this formulation the capacity limits the amount of knowledge that an example can store; problematic points are eventually "sacrificed" in the interest of good generalization. The symmetric convex quadratic programming problem (21) has no local minima, and that, along with the sparsity of the solution, make the SVM a viable and attractive classifier. The weight is given by $\mathbf{w}=\sum_{i} \alpha_{i} y_{i} \mathbf{x}_{i}=\sum_{i \in \mathrm{Sv}} \alpha_{i} y_{i} \mathbf{x}_{i}$, the bias can be computed by applying the Karush-Kuhn-Tucker conditions to the support vectors $[3,10]$, and the machine predicts new cases using

$$
f(\mathbf{x})=\operatorname{sgn}\left(\sum_{i \in \mathrm{SV}} \alpha_{i} y_{i} \mathbf{x}_{i}^{\mathrm{T}} \mathbf{x}+b\right) .
$$

Having protected the generalization ability of the machine we are ready to increase the dimensionality of the space. We use a device that follows from the realization that in both (21) and (22) the data enter the problem only in the form of 
Fig. 12 Two classes of points interspersed, not linearly separable, with $y_{i}=+1$ for the circles and $y_{i}=-1$ for the crosses. Dashed lines show the Gaussian (RBF) potential functions around each point; the solid curve is the sum of those functions, which produces the class separator.

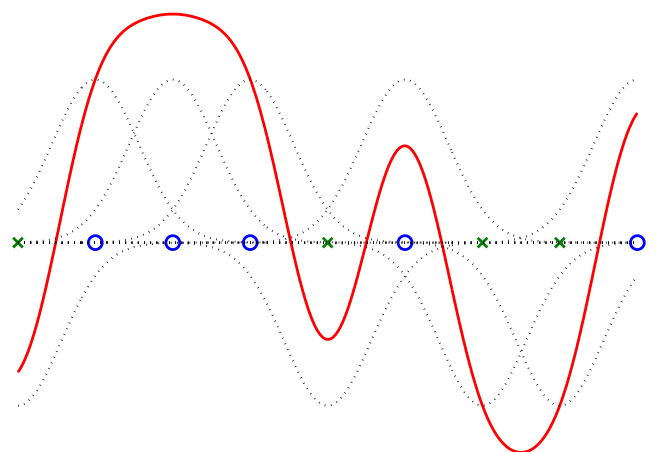

scalar products. It is then possible to have a nonlinear separating surface while still keeping the linearity of the machine by substituting

$$
\mathbf{x}_{1}^{\mathrm{T}} \mathbf{x}_{2} \rightarrow K\left(\mathbf{x}_{1}, \mathbf{x}_{2}\right)=\boldsymbol{\phi}\left(\mathbf{x}_{1}\right)^{\mathrm{T}} \boldsymbol{\phi}\left(\mathbf{x}_{2}\right)
$$

for some mapping $\boldsymbol{\phi}(\mathbf{x})$. A function $K$ that can be so expressed is called a kernel. There is no one-to-one correspondence between mapping and kernel, and, more important, it is not necessary to know $\phi$ to find $K$. The mapping may be into a space of hundreds of thousands of dimensions, yet to gain access to it we only need the much smaller and simpler kernel.

Some kernels stretch out the examples into the added dimensions in such a way that gaps open up between the examples which permit a flat separating surface to pass through. For example, Figure 11 shows the effect of applying to a nonseparable one-dimensional set the mapping $x \rightarrow \boldsymbol{\phi}(x)=\left[\begin{array}{lll}x \sqrt{ } 2 & x^{2} & 1\end{array}\right]^{\mathrm{T}}$, which corresponds to the polynomial kernel $K\left(\mathbf{x}_{1}, \mathbf{x}_{2}\right)=\left(\mathbf{x}_{1}{ }^{\mathrm{T}} \mathbf{x}_{2}+1\right)^{2}$. A very popular alternative, the radial basis function $(\mathrm{RBF})$ kernel

$$
K\left(\mathbf{x}_{1}, \mathbf{x}_{2}\right)=\exp \left(-\left(\mathbf{x}_{1}-\mathbf{x}_{2}\right)^{\mathrm{T}}\left(\mathbf{x}_{1}-\mathbf{x}_{2}\right) / 2 \sigma^{2}\right)
$$

surrounds every point with a (usually Gaussian) surface that resembles a potential function in the sense that it "repels" the separating surface, as shown in Figure 12. The explicit mapping is not known, though in any case in the hyperspace implied by the kernel the separating surface will become flat. The Gaussian width $\sigma$ is an adjustable parameter; the RBF kernel is found to work best when $\sigma$ is on the order of the average separation between points.

One view of the RBF kernel, as well as other alternatives, is that it contains some measure of proximity or similarity between two vectors $\mathbf{x}_{1}$ and $\mathbf{x}_{2}$. The function attains a maximum when $\mathbf{x}_{1}=\mathbf{x}_{2}$ and declines as the points become more distant or dissimilar. (In the case of the polynomial kernel the similarity involves parallelism rather than closeness.) This provides some measure by which the system can determine whether new cases are similar to (i.e., in the same class as) others.

The SVM principle can also be applied to regression problems, where $y_{i} \in \mathbb{R}$ instead of $\{-1,1\}$. The machine creates a surface with a surrounding tube of ad- 

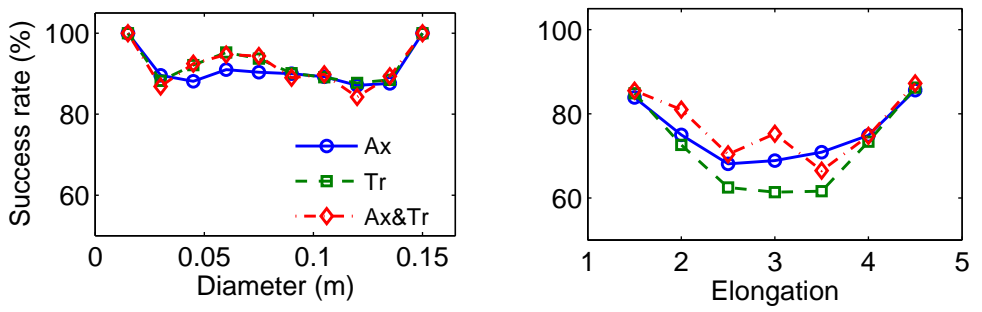

Fig. 13 SVM classification example using dipole moments.

justable width and wiggles it until a given loss function reaches a minimum. The usual choice for this loss function assigns no penalty to points that rest inside the tube and penalizes outliers linearly. The resulting optimization problem is similar to (20): the objective function to be minimized has to strike a balance between fitting accuracy (measured by the capacity and slack variables) and model simplicity (measured by the norm of the weight vector) [10].

Figure 13 shows the results of an SVM classification experiment using synthetic dipole parameters. We choose a range of spheroid diameters (from 1.5 to $15 \mathrm{~cm}$ ) and a range of elongations (from 0.5 to 4.5 ) representative of UXO and generate 1000 spheroids with random diameters and elongations and with conductivities and permeabilities representative of steel and aluminum. We then compute their induced dipole moments under unit axial and transverse uniform primary field excitation using analytic solutions of the EMI equations [1]. Then for given values of the diameter we train an open-source SVM implementation [17] with 200 examples, telling the machine which ones are larger than the given diameter and which ones are not. After that, we test the SVM predictions on the 800 remaining examples. The figure shows the success rate (defined as the number of correct predictions divided by the total number of tests) as a function of increasing cutoff diameter. The second panel displays the results of repeating the experiment using elongation as the classification parameter. Classification is imperfect, but the results are encouraging [11].

A key discrimination quantity of interest to field workers is the size or volume of an unseen object. To motivate use of the SVM, as well as SEA parameters, we note first that the basic dipole parameters do not necessarily correspond in a simple way to volume. Larger objects do not always produce larger dipole values, especially for composite objects observed over a broad band. Dipole responses can be extracted from within the lowest orders of excitation and response in the complete set of $B_{k}^{j}$. Figure 14 shows that both different materials and also different object proportions can reverse the intuitive ordering of the responses in terms of dipole magnitudes. Even while, by contrast, the (reasonably truncated) full set of $B_{k}^{j}$ expresses all possible response behaviors of the objects, there is again no simple, intuitively evident correspondence between the parameter magnitudes that allows ready inference of volume [25]. We need a tool such as the SVM. As a test, an SVM was trained on sets of $B_{k}^{j}$ modal response parameters for spheroids of different shapes, materials, and volumes. These had been sorted into "small" and "large" classes based on a 
Table 1 SVM classification of spheroids based on their response coefficients.

\begin{tabular}{c|cc}
\hline & Predicted Large & Predicted Small \\
\hline True Large & 99 & 1 \\
True Small & 0 & 100 \\
\hline
\end{tabular}

chosen volume cutoff. When the trained algorithm was applied to 200 new ("unknown") cases it classified them as shown in Table 1 [25]. These excellent results suggest that the limitations on classification in Figure 13 are due to the shortcomings of the dipole parameters for classification, not to the SVM itself.

The type of analysis just described becomes problematic when we go to the field because it is difficult to obtain these intrinsic object characteristics from measured data. To find the dipole moments - not entirely consistent discriminators anywaywe first have to determine the target's location and orientation, which results precisely in the nonlinear searches that we want to avoid. The spheroidal coefficients

Fig. 14 Top: Low-frequency modal response magnitudes $B_{k}^{j}$ corresponding to dipole responses to uniform excitation fields in the corresponding directions, for a permeable and a non-permeable spheroid. The smaller object produces larger values. Bottom: Same, but for two spheroids of the same material and with the same volume. With different elongation ratios (1.5 and 2 ) they produce disparate dipole responses at $2 \mathrm{kHz}$.

Fig. 15 SVM regression for depth. The machine uses 800 training examples obtained with the SEA and takes 600 tests, all with normalized inphase and quadrature data at $f=390 \mathrm{~Hz}$ and 14 spatial points. Less than $3 \%$ of the tests (highlighted with circles) have their depth misjudged outside the 5-cm range shown by dim gray lines.
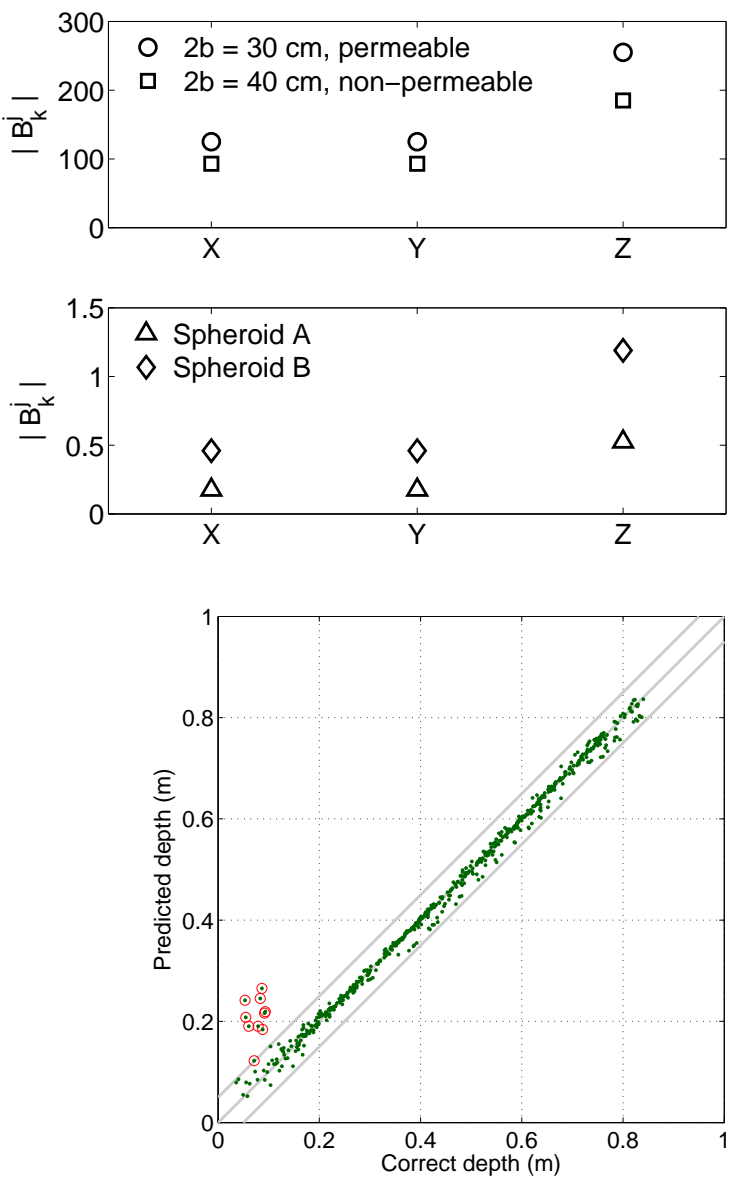
are better discriminators but are nontrivial to determine, even when the location and orientation are known exactly. On the other hand, practitioners in areas like handwriting recognition [2] routinely exploit the statistical, model-independent character of the SVM algorithm and apply it to "raw" data that has not been distilled into simpler or unifying parameters. Encouraged by their results, we have employed the SEA [21] as a dependable and accurate model to generate synthetic secondary fields for a collection of UXO at known depths and then used SVM regression to extract unknown depths for other instances. Figure 15 shows a frequency-domain example. A similar approach has been tried on measured data, with reasonable success [13].

\section{Clutter Reduction by Upward Continuation}

In most realistic situations, subsurface UXO discrimination is clutter-limited. Physical clutter causes signal clutter, which lowers the SNR to a point where signals are unintelligible by any means of processing. This applies particularly to GPR but afflicts EMI as well. Wherever ordnance has failed to explode other ordnance probably has exploded, leaving fragments of metal in or on the soil. As a general, order-ofmagnitude rule of thumb, the magnetic response of a metal body is proportional to its volume. However, fragments are often shallower than a UXO, and thereby nearer the sensor. The $1 / r^{3}$ or $1 / r^{6}$ factors for signal decay cited above can therefore make clutter signals quite strong relative to those from UXO, even while the fragments are inherently much weaker scatterers. If one could observe the scene from a greater elevation, the ratio of distances to UXO and to clutter would become similar, eliminating this problem. While actual sensor elevation is impractical-if only because the overall level of signal might well diminish to the level of the background-the same advantages can sometimes be obtained by computational upward continuation of fields that are measured near the ground.

Our strategy here will be to obtain magnetic-field data over a grid near the surface at some elevation $z_{m}$. The data are then used to infer a sheet of equivalent sources

Fig. 16 Field setup, UXO, clutter, and sensor.

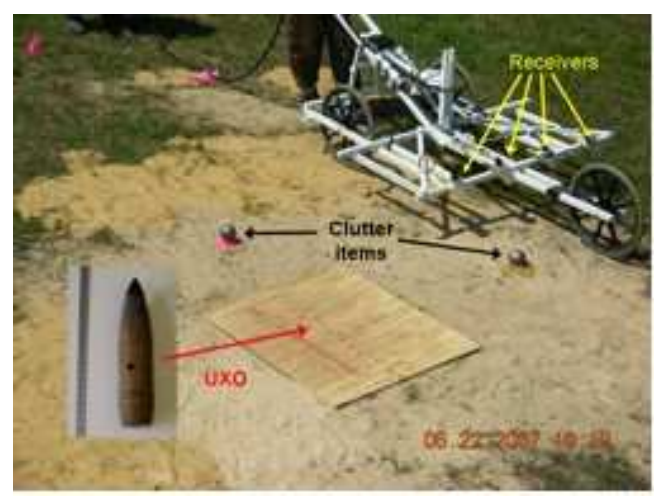


over the ground surface that reproduces the measured values. In particular, given the ê component of $\mathbf{H}$ over a surface, we use the gradient of (11) to solve for $q_{m}$ over a lower surface $S_{0}$ :

$$
H_{e}(\mathbf{r})=\int_{S_{0}} d S^{\prime} q_{m}\left(\mathbf{r}^{\prime}\right) \frac{\hat{\mathbf{e}} \cdot \hat{\mathbf{R}}}{4 \pi R^{2}},
$$

where $\mathbf{R}=\mathbf{r}-\mathbf{r}^{\prime}, \mathbf{r}$ is at elevation $z_{m}$, and $\mathbf{r}^{\prime}$ is on the ground surface. For computational purposes (25) is discretized to form a matrix equation. In this instance, in contrast to assumptions heretofore, let us assume that the object is responding to a single broad primary field while the receivers sample the resulting single secondary field at diverse points (the system depicted in Figure 16, where the survey field is surrounded by a single large transmitter loop [5]). In effect, $H_{e}$ forms a boundary condition on the field above a plane at $z_{m}$. The $q_{m}$ solution produces fields through the integral that satisfy that boundary condition and that also produce an $\mathbf{H}$ field above the boundary that will satisfy all applicable governing equations. Therefore, having obtained the $q_{m}$ from the boundary data via (25), we use it to predict data that would be obtained at a higher elevation. This approach was tested in the field using a buried UXO and shallower pieces of clutter (shotputs, Figure 16).

Figures 17 and 18 show results from this scheme. In the top row, the calculated field on the data plane at $z_{m}$ based on the $q_{m}$ solution at $z=0$ closely reproduces the data. Note that the clutter signals are substantially stronger than those from the UXO. In the bottom row, the signal calculated by continuation to a higher elevation once again matches the corresponding data quite well. While all signals have declined in magnitude, the UXO now produces a response roughly twice that of the clutter. Further, the picture from upward continuation is not afflicted with noise characteristic of the weaker measurements from greater elevation. Projecting the secondary field further upward (Figure 18) shows further and further decrease in the contribution of the clutter and greater prominence of the UXO. This illustration is chosen because the clutter signals are clearly visible alongside the UXO, a situation one might readily deal with otherwise in practice. However, further investigations show this same sort of clutter suppression in the continued fields even when a clutter signal much stronger than the UXO is mixed within it, from an object directly above it [15].

\section{Summary}

To sense subsurface UXO one must detect the metal they contain using electromagnetic devices. Discrimination is difficult because low frequencies and long wavelengths are required for ground penetration and reasonable SNR, which limits resolution. Electromagnetic induction sensors have the advantage of being UWB (10's of Hz up to 10's or perhaps 100's of $\mathrm{kHz}$ ) with the ground essentially transparent to magnetic fields over the entirety of the band. Within metal objects, the fields operate by diffusion. Over the relevant soil and air distances $(<10 \mathrm{~m})$, the fields 

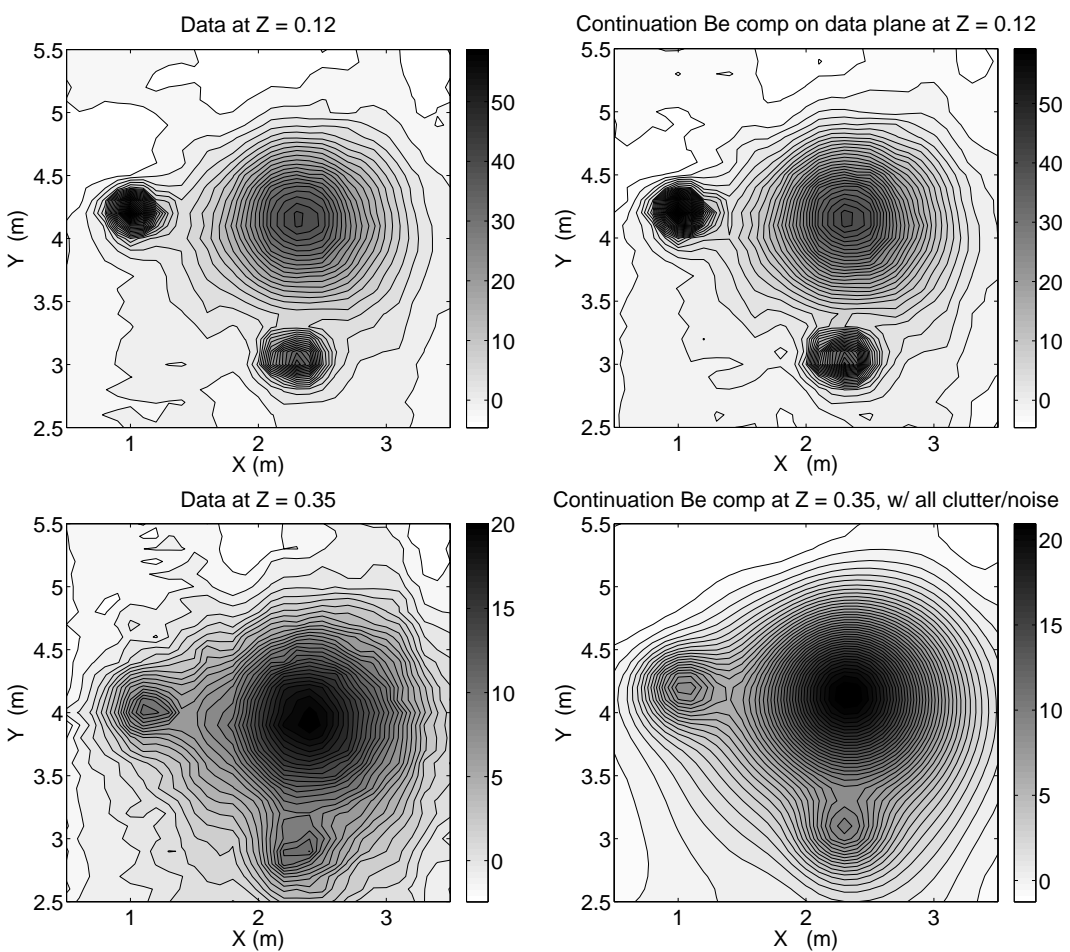

Fig. 17 Case with clutter produced by two shotputs and a 105-mm projectile at the center. Left: data. Right: Continuation results. Top row: Initial elevation. Bottom: signal for sensor at an elevation $23 \mathrm{~cm}$ higher.
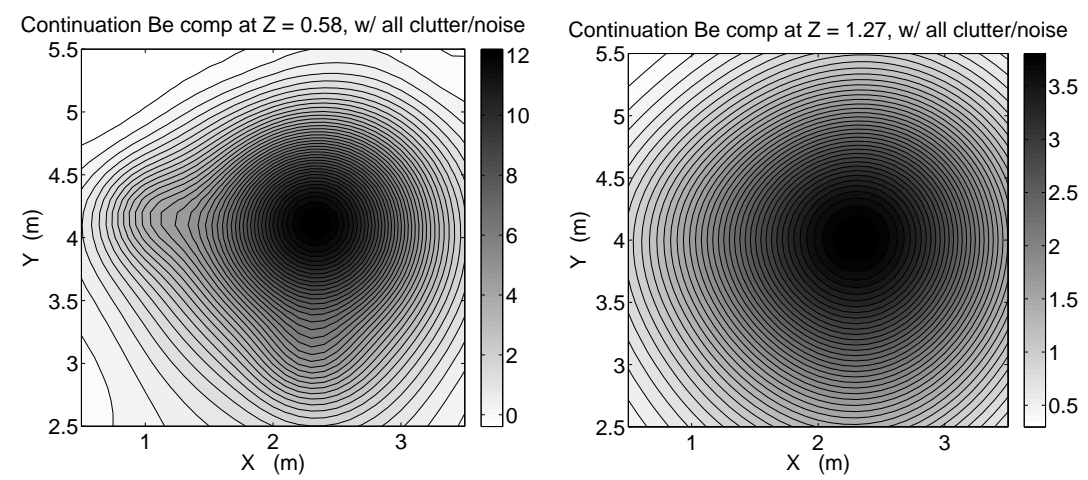

Fig. 18 Computed signals for a $105 \mathrm{~mm}$ UXO plus two surface clutter items, with data continued beyond the $35-\mathrm{cm}$ elevation of the previous figure to $58 \mathrm{~cm}, 81 \mathrm{~cm}$, and $127 \mathrm{~cm}$ (top, middle, bottom). 
can be expressed entirely in terms of a scalar magnetic potential. In our parameter space and scale of observation, there are no waves in the EMI regime, hence no true reflections, diffractions, refractions, or resonances in the wave sense.

One can produce rigorous and fast forward models for EMI responses by chosen metal objects, for known sensors, using the Standardized Excitation Approach. This can be done without solving in detail for the responses within the object. Instead one infers the object's exterior responses to a basis of defined fundamental excitations. Any complete excitation can be expressed as a superposition of the fundamental excitations; therefore any complete response is a corresponding superposition of responses to those excitations. Once the SEA model for an object is defined, its execution is very fast.

For discrimination or classification, the SEA provides highly realistic possible signals in the course of optimizations to match patterns in data from unknown objects. The method can also provide large numbers of high fidelity training examples for statistical learning machines, such as the Support Vector Machine. The SVM operates by implicitly mapping from an original data space to a hyperspace. In the latter the originally intermixed classes for discrimination are separated quite simply by a (hyper)plane. Having trained SVMs using the SEA or analytical solutions, we show some success in using the method to classify objects geometrically, based on their dipole moment parameters; to classify them for size based on sets of their unique SEA parameters themselves; and to estimate their depth using raw signals instead of distilled parameters.

In realistic situations, UXO discrimination is clutter limited. Computational upward continuation shows promise as a physics-based method of EMI clutter suppression. A sheet of equivalent sources is inferred from data at one elevation. These can then predict the signal at greater elevations, at which clutter influences fade.

Acknowledgements We gratefully acknowledge contributions to the work reported here by Dr. Benjamin E. Barrowes, Professor Xudong Chen, Ms. Xiang-Xiang Cheng, Mr. Steven M. Griffin, Professor Shah A. Haider, Mr. Lynn Helms, Professor Keith D. Paulsen, Ms. Irma Shamatava, Professor Fridon Shubitidze, Dr. Keli Sun, Dr. Bae-Ian Wu, Dr. Beijia Zhang, and the late Professor Jin-Au Kong.

\section{References}

1. Barrowes, B.E., O’Neill, K., Grzegorczyk, T.M., Chen, X., Kong, J.A.: Broadband analytical magneto-quasistatic electromagnetic induction solution for a conducting and permeable spheroid. IEEE Trans. Geosci. Remote Sensing 42, 2479-2489 (2004)

2. Boser, B.E., Guyon, I.M., Vapnik, V.N.: A training algorithm for optimal margin classifiers. In: D. Haussler (ed.) Proceedings of the 5th Annual ACM Workshop on Computational Learning Theory, pp. 144-152. ACM Press (1992)

3. Burges, C.J.C.: A tutorial on Support Vector Machines for pattern recognition. Data Mining and Knowledge Discovery 2, 121-167 (1998)

4. Butler, D.K.: Implications of magnetic backgrounds for unexploded ordnance detection. J. Appl. Geophys. 54, 111-125 (2003) 
5. Cattach, M.K., Stanley, J.M., Lee, S.J., Boyd, G.W.: Sub-Audio-Magnetics (SAM): a highresolution technique for simultaneously mapping electrical and magnetic properties. Explor. Geophys. 24, 387-400 (1993). See also http: / /www.g-tek.biz/library.html

6. Chen, C.C., Higgins, M.B., O’Neill, K., Detsch, R.: Ultrawide-bandwidth fully-polarimetric ground penetrating radar classification of subsurface unexploded ordnance. IEEE Trans. Geosci. Remote Sensing 39, 1221-1230 (2001)

7. Chen, X., O’Neill, K., Barrowes, B.E., Grzegorczyk, T.M., Kong, J.A.: Application of a spheroidal mode approach with differential evolution in inversion of magnetoquasistatic data for UXO discrimination. Inv. Prob. 20, 27-40 (2004)

8. Chen, X., O'Neill, K., Grzegorczyk, T.M., Kong, J.A.: Spheroidal mode approach for the characterization of metallic objects using electromagnetic induction. IEEE Trans. Geosci. Remote Sensing 45, 697-706 (2007)

9. Cheng, D.K.: Field and Wave Electromagnetics. Addison-Wesley, Reading, MA (1989)

10. Cristianini, N., Shawe-Taylor, J.: An Introduction to Support Vector Machines and other Kernel-Based Learning Methods. Cambridge University Press, Cambridge (2000)

11. Fernández, J.P., Barrowes, B., O’Neill, K., Paulsen, K., Shamatava, I., Shubitidze, F., Sun, K.: Evaluation of SVM classification of metallic objects based on a magnetic-dipole representation. In: J.T. Broach, R.S. Harmon, J.H. Holloway Jr. (eds.) Detection and Remediation Technologies for Mines and Minelike Targets XI, Proceedings of SPIE, vol. 6217, pp. 621703. Bellingham, WA (2006)

12. Fernández, J.P., Barrowes, B., O’Neill, K., Shamatava, I., Shubitidze, F., Sun, K.: A dataderived time-domain SEA for UXO identification using the MPV sensor. In: R.S. Harmon, J.T. Broach, J.H. Holloway Jr. (eds.) Detection and Sensing of Mines, Explosive Objects, and Obscured Targets XIII, Proceedings of SPIE, vol. 6953, pp. 6953-1H. Bellingham, WA (2008)

13. Fernández, J.P., Sun, K., Barrowes, B., O’Neill, K., Shamatava, I., Shubitidze, F., Paulsen, K.: Inferring the location of buried UXO using a Support Vector Machine. In: R.S. Harmon, J.T. Broach, J.H. Holloway Jr. (eds.) Detection and Remediation Technologies for Mines and Minelike Targets XII, Proceedings of SPIE, vol. 6553, pp. 6553-0B. Bellingham, WA (2007)

14. O'Neill, K.: Ultra-wideband, fully polarimetric ground penetrating radar for UXO discrimination. ESTCP Final Technical Report, Project 199902. www . estcp. org (2005)

15. O'Neill, K.: Processing for clutter evasion in UXO discrimination. SERDP Project MM-1590 Final Technical Report. www . serdp.org (2008)

16. Pasion, L.R., Oldenburg, D.W.: A discrimination algorithm for UXO using time domain electromagnetics. J. Environ. Eng. Geophys. 6, 91-102 (2001)

17. Rüping, S.: mySVM-Manual. University of Dortmund, Lehrstuhl Informatik 8 (http:// wwW-ai.cs.uni-dortmund.de/SOFTWARE/MYSVM/) (2000)

18. Shubitidze, F., O’Neill, K., Barrowes, B., Shamatava, I., Sun, K., Fernández, J.P., Paulsen, K.: Application of the normalized surface magnetic charge model to UXO discrimination in cases with overlapping signals. J. Appl. Geophys. 61, 292-303 (2007)

19. Shubitidze, F., O'Neill, K., Shamatava, I., Sun, K., Paulsen, K.D.: Fast and accurate calculation of physically complete EMI response by a heterogeneous metallic object. IEEE Trans. Geosci. Remote Sensing 43, 1736-1750 (2005)

20. Stratton, J.A.: Electromagnetic Theory. McGraw-Hill, New York (1941)

21. Sun, K., O’Neill, K., Shubitidze, F., Shamatava, I., Paulsen, K.: Fast data-derived fundamental spheroidal excitation models with application to UXO discrimination. IEEE Trans. Geosci. Remote Sensing 43, 2573-2583 (2005)

22. Vapnik, V.N.: An overview of statistical learning theory. IEEE Trans. Neural Netw. 10, 988999 (1999)

23. Wait, J.R.: A conducting sphere in a time varying magnetic field. Geophysics 16, 666-672 (1951)

24. Zhang, B.: Classification, identification, and modeling of unexploded ordnance in realistic environments. Ph.D. thesis, Massachusetts Institute of Technology (2008)

25. Zhang, B., O’Neill, K., Kong, J.A., Grzegorczyk, T.M.: Support vector machine and neural network classification of metallic objects using coefficients of the spheroidal MQS response modes. IEEE Trans. Geosci. Remote Sensing 46, 159-171 (2008) 
26. Zhang, B., O'Neill, K., Wu, B.I., Kong, J.A.: Absolute calibration of EMI measurements and application to soil magnetic susceptibility inference. J. Environ. Eng. Geophys. (2008). In press 